ISSN: 2550 - 682X

DOI: $10.23857 /$ pc.v2i10.375

Recepción: 31 / 01 / 2017

Aceptación: 19/ 05 / 2017

Publicación: 31 / 10 / 2017

Ciencias de la educación

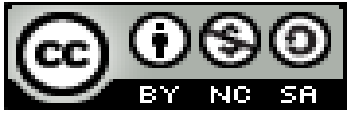

Artículo de investigación

\title{
Motivación como factor clave en la praxis gerencial del docente universitario
}

\section{Motivation as a key factor in the managerial practice of the university teacher}

\section{Motivação como fator chave na prática gerencial do professor universitário}

\author{
Homero M. Ferrín-Schettini ${ }^{\mathrm{I}}$ \\ homeromanuelferrin@hotmail.com \\ Rubén D. Mero-Mero II \\ rdmero111@hotmail.com \\ María J. Alcívar-Mero III \\ jessenialcivar@hotmail.com
}

Correspondencia: homeromanuelferrin@hotmail.com

I Diploma Superior en Economía Internacional, Diploma Superior en Economía del Ecuador y del Mundo, Diploma Superior en Gestión Empresarial Internacional, Magister en Negocios Internacionales y Gestión de Comercio Exterior, Ingeniero Civil, Arquitecto, Docente de la Universidad Laica Eloy Alfaro de Manabí, Manta, Ecuador.

II Diploma Superior en Economía del Ecuador y del Mundo, Magister en Negocios Internacionales y Gestión de Comercio Exterior, Ingeniero Comercial, Ingeniero en Contabilidad y Auditoría, Docente de la Universidad Laica Eloy Alfaro de Manabí, Manta, Ecuador.

III Magister en Contabilidad y Auditoría, Especialista en Diseño Curricular por Competencias, Ingeniera Comercial, Docente de la Universidad Laica Eloy Alfaro de Manabí, Manta, Ecuador. 


\section{Resumen}

Dentro del contexto educativo la figura del docente universitario es fundamental para lograr la formación de profesionales que impulsarán el desarrollo de un país, éste debe ser un guía, orientador, investigador, facilitador de oportunidades que va a contribuir con el proceso de enseñanza-aprendizaje, fomentando la utilización de estrategias metodológicas, objetivos, métodos, recursos, evaluación, orientadas a estimular las actividades educativas con base en las necesidades e inquietudes del estudiante para que así este logre su desarrollo físico-intelectual y moral. Además de las actividades de docencia, el profesor universitario debe cumplir con otras actividades que forman parte de sus funciones laborales como la extensión, investigación, la autoformación, así como también una actividad de gran complejidad como lo es la gerencia. El docente universitario en su función de gerente debe dirigir sus acciones a orientar, evaluar, controlar y organizar el quehacer educativo, desempeñando un papel muy importante como ejecutor de las políticas educativas que promuevan cambios importantes que induzcan a mejorar la calidad de la educación. Uno de los problemas más graves de la gerencia en la educación universitaria, es la efectividad con que la institución alcanza sus objetivos y satisface la necesidad de la sociedad, y que depende del desempeño del trabajo que realice el gerente. Sin embargo, se han encontrado casos de gerentes universitarios que han demostrado ser exitosos en su gestión, fundamentado en la forma como han alcanzado sus objetivos y satisfecho las necesidades de una institución universitaria, en su proyección hacia la sociedad, en su trabajo del día a día; en fin, en todas las acciones realizadas para que la organización educativa logre sus metas. No obstante, el docente universitario no siempre cuenta con las condiciones favorables para llevar con éxito el proceso gerencial. Lo anteriormente expuesto se debe a factores intrínsecos y extrínsecos que pueden afectar significativamente la motivación de un docente siendo susceptibles a ser analizados para generar numerosas investigaciones orientadas a las posibles soluciones que puedan mejorar el desempeño gerencial del docente universitario. De allí que el presente ensayo tiene como propósito develar algunas de las dificultades que presenta el docente universitario en su praxis gerencial afectando un aspecto muy importante del ser humano como lo es la motivación, pero a su vez vislumbrar algunas sugerencias orientadas a contribuir en la disminución de éstos factores de origen personal e institucional revirtiendo el proceso de desmotivación. 
Palabras clave: motivación; gerencia universitaria; educación superior; universidad.

\section{Abstract}

Within the educational context the figure of the university teacher is fundamental to achieve the training of professionals that will drive the development of a country, it must be a guide, counselor, researcher, facilitator of opportunities that will contribute to the teaching-learning process, promoting the use of methodological strategies, objectives, methods, resources, evaluation, aimed at stimulating educational activities based on the needs and concerns of the student so that this will achieve their physical-intellectual and moral development. In addition to the teaching activities, the university professor must comply with other activities that are part of their job functions such as extension, research, self-training, as well as a highly complex activity such as management. The university teacher in his role as manager must direct their actions to guide, evaluate, control and organize the educational work, playing a very important role as executor of educational policies that promote important changes that lead to improve the quality of education. One of the most serious problems of management in university education is the effectiveness with which the institution achieves its objectives and satisfies the need of society, and that depends on the performance of the work performed by the manager. However, there have been cases of university managers who have proven to be successful in their management, based on the way they have achieved their objectives and satisfied the needs of a university institution, in their projection towards society, in their work of the day day; in short in all the actions carried out so that the educational organization achieves its goals. However, the university teacher does not always have the favorable conditions to successfully carry out the management process. The foregoing is due to intrinsic and extrinsic factors that can significantly affect the motivation of a teacher being susceptible to be analyzed to generate numerous research oriented to possible solutions that can improve the managerial performance of the university teacher. Hence, the purpose of this essay is to reveal some of the difficulties that the university teacher presents in his managerial praxis affecting a very important aspect of the human being as it is the motivation, but at the same time to glimpse some suggestions aimed at contributing to the decrease of these factors of personal and institutional origin reversing the demotivation process.

Keywords: Motivation; university management; higher education; university. 


\section{Resumo}

No contexto educacional, a figura do professor universitário é fundamental para a formação de profissionais que impulsionarão o desenvolvimento de um país, deve ser um guia, conselheiro, pesquisador, facilitador de oportunidades que contribuam para o processo ensino-aprendizagem, promovendo o uso de estratégias metodológicas, objetivos, métodos, recursos, avaliação, com o objetivo de estimular atividades educacionais com base nas necessidades e preocupações do aluno, para que isso alcance seu desenvolvimento físico-intelectual e moral. Além das atividades de ensino, o professor da universidade deve cumprir outras atividades que fazem parte de suas funções de trabalho, tais como extensão, pesquisa, auto-treinamento, bem como uma atividade altamente complexa, como a gestão. O professor da universidade em seu papel de gerente deve dirigir suas ações para orientar, avaliar, controlar e organizar o trabalho educacional, desempenhando um papel muito importante como executor de políticas educacionais que promovam mudanças importantes que levem a melhorar a qualidade da educação. Um dos problemas mais sérios de gestão na educação universitária é a eficácia com a qual a instituição alcança seus objetivos e satisfaz as necessidades da sociedade, e isso depende do desempenho do trabalho realizado pelo gerente. No entanto, houve casos de gerentes universitários que provaram ser bem sucedidos em sua gestão, com base na maneira como eles alcançaram seus objetivos e satisfez as necessidades de uma instituição universitária, na sua projeção para a sociedade, no seu trabalho do dia dia em suma, em todas as ações realizadas para que a organização educacional alcance seus objetivos. No entanto, o professor universitário nem sempre tem as condições favoráveis para realizar com sucesso o processo de gestão. O que precede é devido a fatores intrínsecos e extrínsecos que podem afetar significativamente a motivação de um professor sendo suscetível a ser analisado para gerar inúmeras pesquisas orientadas para possíveis soluções que possam melhorar o desempenho gerencial do professor da universidade. Assim, o objetivo deste ensaio é revelar algumas das dificuldades que o professor da universidade apresenta em sua práxis gerencial afetando um aspecto muito importante do ser humano, pois é a motivação, mas ao mesmo tempo vislumbrar algumas sugestões que visam contribuir para a diminuição desses fatores de origem pessoal e institucional que invertem o processo de desmotivação.

Palavras chave: Motivação; gestão da universidade; educação superior; universidade. 


\section{Introducción}

Dentro del contexto educativo, la figura del docente universitario es fundamental para lograr la formación de profesionales que impulsen el desarrollo de un país, éste debe ser un guía, orientador, investigador, facilitador de oportunidades que va a contribuir con el proceso de enseñanza-aprendizaje, fomentando la utilización de estrategias metodológicas, objetivos, métodos, recursos, evaluación, orientadas a estimular las actividades educativas con base en las necesidades e inquietudes del estudiante para que así este logre su desarrollo físico-intelectual y moral.

Además de las actividades de docencia, el profesor universitario debe cumplir con otras actividades que forman parte de sus funciones laborales como la extensión, investigación, la autoformación, así como también una actividad de gran complejidad como lo es la gerencia, tal como lo señala Lemus (1975), "... el docente no puede ser un simple transmisor de conocimientos, dador de clases o expositor de hechos y teorías, debe, por el contrario, actuar como un elemento propiciador y facilitador de hechos educativos..." (p.216). El docente universitario en su función de gerente debe dirigir sus acciones a orientar, evaluar, controlar y organizar el quehacer educativo, desempeñando un papel muy importante como ejecutor de las políticas educativas que promuevan cambios importantes que induzcan a mejorar la calidad de la educación.

En este sentido, el docente universitario como gerente administrador, debe cumplir ciertas funciones para lograr las metas pre-establecidas en la organización educativa, funciones tales como: planificador, organizador, coordinador de recursos materiales y humanos, lograr la eficacia de estas funciones, de todos y cada uno de sus subordinados dentro de la institución. De igual manera, debe asumir la función de dirigir al personal docente, administrativo y obrero según sea el cargo a desempeñar, creando una atmósfera adecuada y de ese modo ayudarlos a dar sus mejores esfuerzos.

Asimismo, el docente universitario debe ejercer el control dentro de la administración, porque un gerente es un factor fundamental para evitar problemas de conflictos dentro de la institución, es un ente que permite un equilibrio del clima de la organización; el control significa asegurar que la 
organización siga la dirección correcta en la obtención de sus metas, destacando que la responsabilidad que tiene el gerente de la institución universitaria es de tal magnitud, que aún sin querer puede ser el responsable de la infinidad de problemas que se susciten en estos recintos educativos, los gerentes universitarios son responsables de la calidad del proceso y deben asumir la responsabilidad de los resultados, él debe delegar responsabilidades a las personas a su cargo, con cierta autoridad, para lograr el éxito en el cumplimiento de los objetivos propuestos; a éste le compete verificar el cumplimiento de las tareas por cada ente de la organización.

Igualmente, a los gerentes universitarios se les evalúa atendiendo a la eficacia con que coordinen la organización de dichas tareas, son responsables por las acciones de sus subordinados, el éxito o el fracaso de estos últimos es un reflejo directo del éxito o fracaso de los gerentes; todos los miembros de una organización, particularmente la institución universitaria, influyen en los que no pertenecen a la categoría de gerencia, pero todos son responsables de las tareas en particular, la diferencia radica en que los gerentes son los responsables natos, no sólo por su propio trabajo sino también por el trabajo de los demás.

Es por ello y parafraseando a Cárdenas (1995), que uno de los problemas más graves de la educación universitaria pudiera estar representado en la gerencia, la efectividad con que una organización alcanza sus objetivos y satisface la necesidad de la sociedad, depende del desempeño del trabajo que realice el gerente. Sin embargo, se han encontrado casos de gerentes universitarios que han demostrado ser exitosos en su gestión, fundamentado en la forma como han alcanzado sus objetivos y satisfecho las necesidades de una institución universitaria, en su proyección hacia la sociedad, en su trabajo del día a día; en fin, en todas las acciones realizadas para que la organización educativa logre sus metas.

De allí que éste proceso de orientación administrativa como lo es la gerencia universitaria, debe contar con las condiciones necesarias de organización, estructura, humanas, tecnológicas y ambiente para llevarse a cabo con eficacia y eficiencia, según Drucker (1968), "el desempeño de un gerente puede medirse a partir de dos conceptos: eficiencia y eficacia. Ambos elementos contribuyen al efectivo y eficiente desempeño administrativo" s/p. No obstante, el docente universitario no siempre cuenta con las condiciones favorables para llevar con éxito el proceso gerencial. 
Lo anteriormente expuesto se debe a factores intrínsecos y extrínsecos que pueden afectar significativamente la motivación de un docente siendo susceptibles a ser analizados para generar numerosas investigaciones orientadas a las posibles soluciones que puedan mejorar el desempeño gerencial del docente universitario. De allí que el presente ensayo tiene como propósito develar algunas de las dificultades que presenta el docente universitario en su praxis gerencial afectando un aspecto muy importante del ser humano como lo es la motivación, pero a su vez vislumbrar algunas sugerencias orientadas a contribuir en la disminución de éstos factores de origen personal e institucional revirtiendo el proceso de desmotivación.

\section{Gerencia en el contexto educativo}

La gerencia educativa se concibe como el proceso de dirección de esfuerzo humano para lograr funciones y objetivos con participación, compromiso y toma de decisiones eficaces en la institución, con la mejor utilización de los recursos disponibles. Desde este punto de vista, Manes (2005) define la gerencia educativa como "el proceso de conducción de una institución educativa por medio del ejercicio de un conjunto de habilidades directivas orientadas a planificar, organizar, coordinar y evaluar..." (p 3) acciones estratégicas a través de actividades oportunas para alcanzar eficacia en los aspectos pedagógicos, administrativos y comunitarios.

Para ello, se requiere de gerentes capaces de responder a las exigencias estructurales y socioculturales configurándose eficazmente en un sistema que aprende enfrentando problemáticas que el entorno le demanda, es decir, aprenden a través de los cambios sistemáticos que en la institución se producen, lo cual no se da de forma improvisada, requiere de directrices, creatividad, criterios, valores y normas que orienten el desempeño gerencial de forma organizada.

En este sentido, se define Gerencia Educativa como las acciones que los lideres aplican para guiar a los actores educativos hacia una dirección deseada, comprometiéndolas al cambio mediante la solución de sus problemas (Cornejo, 2000). Por su parte, Macha op cit, aborda la gerencia educativa con un sentido de integralidad, es decir, como el proceso de conducción de las organizaciones hacia el logro de resultados que ayuden a realizar acciones de manera planificada, dinámica y con fuerza de cambio. 
Por otro lado, Drucker, P. op. Cit. establece que "gerenciar significa hacer las cosas bien" (p120), se pudiera pensar que la existencia de ineficiencia en una gestión, es ocasionado por el poco esfuerzo y responsabilidad dedicado por el docente a la realización de las actividades requeridas para la óptima realización de las actividades previstas, sin embargo la poca eficiencia en un proceso gerencial puede transcender al mero hecho del esfuerzo y traducirse en la poca motivación que puede sentir un gerente educativo al transitar el camino de la administración.

\section{Dificultades en los procesos gerenciales del contexto universitario}

Entre algunas de las dificultades que puede encontrar el docente universitario en su praxis gerencial y que influyen en su motivación se encuentran primeramente aquellas de naturaleza individual e intrínseca:

- Algunos docentes no cuentan con la preparación académica necesaria en el área gerencial que les proporcione las herramientas adecuadas para desempeñarse en éste tipo de actividad. El docente en su formación académica de pregrado cursa pocas asignaturas relacionadas a la gerencia, no siendo ésta su principal área de conocimiento. Asimismo, no recibe una preparación gerencial inherente al cargo que va a desempeñar dejándose a la experiencia del ensayo y error (empirismo) el cumplimiento de sus funciones. Presentan entre otros elementos inconvenientes en la capacidad de comunicación, toma de decisiones y liderazgo en su relación con otros docentes. En este orden de ideas parafraseando a Chiavenato (1989), se puede afirmar que la formación gerencial es una herramienta eficaz para que el gerente desarrolle habilidades para crear y aplicar estrategias administrativas efectivas, propiciar cambios en su organización y, en general, mejorar su comportamiento gerencial y de ésta manera superar las dificultades propias de la gerencia.

- Para otros docentes universitarios las funciones gerenciales no se encuentran dentro de las actividades de su preferencia por lo que sienten cierta aversión ante la obligación que le impone el Estatuto para el Personal Docente de ejercer un cargo gerencial.

Por otro lado, se encuentran aquellos factores de orden extrínseco o institucional: 
-Los reglamentos o normativas presentan algunos vacíos en los procesos administrativos requeridos para resolver casos de incumplimiento, así como las funciones o tareas no se encuentran bien definidas lo que afecta los objetivos planteados. Los reglamentos que rigen un espacio gerencial educativo no se encuentran correctamente elaborados por lo que afecta la toma de decisiones del docente en la solución de un problema. Al respecto, Kliksberg (1988), "que, en materia de gerencia social, se deben llevar a cabo estilos gerenciales particulares a cada situación, en donde el diseño, la toma de decisión y la acción corresponda con el momento, lo que requerirá de una constante renovación". (p.35 y 97).

- Se evidencia poca motivación de algunos docentes para el cumplimiento de sus funciones y en el apoyo de iniciativas que fortalezcan la organización. El clima organizacional no siempre es favorable para el desempeño gerencial de un docente. El monitoreo, supervisión y evaluación no siempre es bien recibida por parte del colectivo docente. Siendo necesario recordar que la gerencia, como actividad humana, tiene que ver con relación a la dinámica entre el líder y el grupo, es decir, es de la competencia del gerente educativo, al ejercer su liderazgo, la aplicabilidad de sus conocimientos y habilidades, para poder influir en el grupo con el cual interactúan en la solución de los problemas, de igual manera de haber una correspondencia empática del grupo hacia el gerente en la búsqueda de un mismo fin, Al respecto, Hersey y Blanchard (1977), plantean que el liderazgo es el proceso de ejercer influencia sobre un individuo o grupo de individuos en los esfuerzos para la realización de los objetivos en determinada situación.

-Los recursos financieros y materiales en algunos momentos son escasos y los medios tecnológicos presentan numerosas fallas, afectando el proceso gerencial ya que la función administrativa que desempeña el gerente educativo diariamente, debe estar acorde con las innovaciones que continuamente se producen en el ámbito de la administración en general, de tal manera que puedan producirse cambios en todas las instancias personales, grupales y organizacionales. Se nombraron algunas entre otras tantas dificultades que puede encontrar el docente universitario en su praxis gerencial. 


\section{Importancia de la motivación en la praxis gerencial del docente universitario}

Para configurar el sistema educativo de la universidad contemporánea, y tomando en cuenta la importancia de la gerencia en la universidad como un elemento de motivación en la praxis educativa en este nivel es importante señalar que, la universidad es uno de los conceptos más trabajados en la humanidad y en cada momento histórico ha tenido implicaciones importantes para este universo que cada día intenta verterse hacia sí mismo con la finalidad de reencontrase y reinventarse, todos los cambios que se han dado en la universidad desde la antigüedad hasta la fecha, favorecen ineluctablemente a todas aquellas personas que hacen vida en el ámbito universitario. Según Castellanos (2011):

La universidad es una institución social que ha sido, y sigue siendo, un espacio privilegiado de prácticas sociales organizadas alrededor de la creación, recreación, comunicación y difusión de saberes y conocimientos; un tejido de relaciones sociales, científicas, culturales, políticas, económicas, éticas y estéticas, - entre otras - articuladas con la estructura de la sociedad que la contiene, por ello, la composición social, cultural y política de esta institución en determinados momentos históricos condiciona las respuestas de la mayoría de su comunidad a los cambios que se construyen e impulsan en su entorno, y requieren de ella, reacomodos, reformas o transformaciones profundas que toquen las concepciones que están en la base de la idea de universidad (p.25).

Partiendo de lo anterior, es importante destacar que, uno de los aspectos importantes para una educación de calidad en los diferentes niveles del sistema educativo y en especial en la universidad es el componente motivacional, no solo el que pueda tener el estudiantes para cumplir con sus metas, también es necesario señalar que toda la comunidad universitaria debe tener altos niveles de motivación para el logro de un proceso efectivo, es por esto que, el docente universitario como parte de esta comunidad debe procurar reencontrarse con todos aquellos aspectos que le condujeron a especializarse en la docencia y particularmente en la docencia universitaria.

De allí que, Robbins (2004) define la motivación como procesos que fomentan la intensidad, dirección y persistencia del esfuerzo de un individuo por conseguir una meta. Dicha definición 
contempla tres elementos fundamentales. La intensidad consiste en cuánto se esfuerza una persona, lo cual debe canalizarse en una dirección que genere beneficios, por lo que hay que considerar tanto la calidad del esfuerzo como su intensidad. El esfuerzo a utilizar es el que se dirige hacia las metas previstas y es congruente con ellas. Además, la motivación tiene una inclinación a la persistencia, que es la medida de cuánto tiempo sostiene una persona su esfuerzo. Los individuos motivados se aferran a una tarea para alcanzar la meta. La motivación consiste en una necesidad o deseo específico que activa al organismo y dirige la conducta hacia una meta.

Además, Robbins y Coulter (2005) exponen la teoría contemporánea de las tres necesidades de McClelland sobre motivación, con un grado aceptable de apoyo de investigación válida, la cual afirma que dichas necesidades son adquiridas, no innatas y constituyen motivos importantes en la conducta de las personas. También refiere que las necesidades humanas se aprenden durante la vida de las personas siendo el medio educativo un contexto importante para la implementación de procesos que contribuyan al desarrollo de la motivación. Gran parte del interés en estos modelos de motivación se generó a partir de la investigación de McClelland de la Universidad de Harvard. Este autor desarrolló una clasificación que subraya tres de los más dominantes impulsos y señaló su significado para la motivación. Sus estudios revelaron que los impulsos motivadores de las personas reflejaban los elementos de la cultura en la que había crecido: su familia, escuela, iglesia y libros. En la mayoría de las naciones uno o dos de los patrones de motivación tendían a estar muy arraigados entre las personas, porque habían crecido en ambientes similares.

Entre tanto, Newstrom (2007) señala que la gente tiende a desarrollar ciertos impulsos motivadores como producto del ambiente cultural en que vive, los cuales afectan la forma en que los individuos enfocan su vida. Este autor, refiere que la motivación mueve al docente universitario a comportarse, a pensar y sentir en la forma en que lo hacen, siendo el comportamiento motivado, activado, dirigido y sostenido. Igualmente plantea que consisten en necesidades y deseos que se aprenden a través de la experiencia con el mundo social.

Partiendo de lo anterior es importante señalar, que el profesor universitario debe contar con las condiciones y herramientas gerenciales requeridas que le permitan adaptarse a todos los cambios paradigmáticos producto de la globalización y que por ende contribuya a cristalizar las metas de su institución educativa ya que de éstas dependen la calidad de la educación, el clima

\section{4}

Pol. Con. (Edición núm. 12) Vol. 2, No 10, octubre 2017, pp. 14-28, ISSN: 2550 - 682X 
organizacional y otros aspectos de índole gerencial importantes en el funcionamiento de una organización, entendiendo por otro lado, que los estudiantes de este milenio representan una generación de avanzada que tienen acceso a la información y que dominan las redes en algunos casos hasta mejor que el docente. Sin embargo, es importante que el docente tenga un alto grado de motivación en su quehacer diario en la institución en sus funciones gerenciales ya que además de los motivos antes expuestos se vienen suscitando desde el siglo pasado cambios en las universidades que inciden en la planeación y el desarrollo de las mismas:

a. Los cambios externos, que ocurren fuera de la universidad y en los cuales se refleja el incremento de la complejidad del medio ambiente socioeconómico

b. Los cambios internos, que van a reflejar una vida interna de las universidades cada vez más complejas, y que deben abordar nuevas tareas para satisfacer nuevas necesidades, intereses y demandas

c. La redefinición de las relaciones entre la universidad y la sociedad, en la cual las universidades muestran e incrementan su rol socioeconómico

\section{Posibles soluciones orientadas a la motivación del docente-gerente universitario}

Debido a la gran cantidad de cargos gerenciales que se llevan a cabo en las universidades, es necesario realizar cursos de capacitación teórico práctica en gestión educativa-administrativa para que los docentes universitarios adquieran las herramientas o conocimientos necesarios según el cargo gerencial que va a desempeñar, y valorar a través de test u otro tipo de instrumento las capacidades gerenciales y nivel de disposición que posee el, a fin de considerar la posibilidad de que un profesional de la educación ejerza o no un cargo de índole gerencial y de ser definitivo asegurar un buen desempeño y altos niveles de motivación en una actividad tan compleja.

Por otra parte, realizar jornadas anualmente encaminadas a una revisión exhaustiva de los reglamentos o normativas para complementar los vacíos o deficiencias que puedan presentar y que faciliten la toma de decisiones en los diferentes casos que se puedan suscitar, así como también para el eficaz y eficiente cumplimento de las tareas o funciones que se deben llevar a cabo dentro de una organización educativa. Asimismo, dotar de los recursos materiales y 
humanos que se requieren para que cada docente pueda cumplir sus funciones considerando además el adecuado mantenimiento para que los equipos electrónicos funcionen eficientemente principalmente en los tiempos actuales en que la tecnología ha pasado ocupar una importante herramienta de trabajo para los docentes universitarios.

\section{Conclusión}

El docente en el campo universitario debe desempeñar diversos roles como parte de sus funciones laborales, entre ellas una de las más complejas siempre ha sido la gerencia, y para ejercerla eficiente y eficazmente, éste debo contar con altos niveles de motivación y preparación que le permitan llegar al cumplimiento de las metas propuestas en su organización educativa, no obstante el docente universitario debe enfrentar una serie de obstáculos que dificulta un buen desempeño en el cargo gerencial asumido.

En este sentido, los recintos universitarios deben crear las condiciones necesarias de organización, estructura, humanas, tecnológicas y ambiente inherentes al cargo gerencial que el docente va a desempeñar, desde la correcta elaboración de sus normativas, preparación en el área gerencial, hasta la dotación de recursos materiales y humanos necesarios para llevar a cabo eficientemente sus funciones.

De tal manera, que el docente universitario sea capaz de convertir en propia y con alta responsabilidad las metas encomendadas, procurando el enaltecimiento y humanización de todos a través de un clima organizacional favorable. Este profesional debe poseer una capacidad de comprensión de la realidad, debe basarse en un profundo respeto humano, ética profesional, capacidad para tomar decisiones y sentido común para saber cuándo debe asumir la responsabilidad y cuándo la debe delegar; debe, además, conocer la incidencia de ésta sobre el individuo y grupos, así como la proyección social de la institución hacia la comunidad.

Contar con un docente universitario con altos niveles de motivación y capacitado en el área gerencial puede garantizar el buen funcionamiento del quehacer educativo de las universidades, pero sobre todo favorecer directa o indirectamente el fin último de la educación superior, egresar excelentes profesionales que puedan cubrir las demandas sociales-políticas de una nación. En fin, 
la gerencia es una parte principal del proceso educativo; le otorga el toque final de excelencia que ofrece tanto la filosofía educativa, la estructura y la cultura organizacional; y finalmente la calidad del educador desde su origen y durante todo el proceso educativo.

\section{Referencias bibliográficas}

Cárdenas, L. (1995). Plan de acción. Ministerio de Educación. 15 de Enero. Caracas. Venezuela.

Castellano, M. (2011). La universidad en el siglo XXI. Caracas: Universidad Católica Andrés Bello.

Chiavenato, I. (2003). Introducción general a la administración. Tercera Edición. México: Editorial Mc Graw-Hill.

Cornejo, R. (2000). Avances actuales en el entendimiento de la inteligencia. Apuntes de Clase. Santiago-Universidad de Chile: Equipo de Psicología Educacional.

Drucker, P. (1968). Gerente eficaz. Rio de Janeiro, Brasil.

Hersey, P. y Blanchard, K. (1977). La teoría del ciclo vital del liderazgo. México: Editorial Interamericana.

Klisber, R. (1988). Hacia un nuevo paradigma en gestión pública. Investigación y Gerencia.

Manes. J. M. (2005). Gestión Estratégicas para Instituciones Educativas. Buenos Aires Argentina: Ediciones Granica.

Newstrom, J. (2007). Comportamiento humano en el trabajo. México: McGraw Hill Interamericana.

Lemus, L. (1975). Administración, Dirección y Supervisión de Escuelas. Buenos Aires. Argentina. Editorial Kapelusz.

Robbins, S. (2004). Comportamiento organizacional. México: Pearson Educación. 
Robbins, S. y Coulter, M. (2005). Administración. México: Pearson Educación. 